\title{
A SVD BASED SCHEME FOR POST PROCESSING OF DCT CODED IMAGES
}

\author{
Vinay Kumar Srivastava \\ Assistant Professor, Department of Electronics and Communication Engineering, \\ Motilal Nehru National Institute of Technology, Allahabad, India \\ vks.mnnit@gmail.com,vksrivastava12@rediffmail.com
}

Received 8th May 2008; accepted 28th September 2009

\begin{abstract}
In block discrete cosine transform (DCT) based image compression the blocking artifacts are the main cause of degradation, especially at higher compression ratio. In proposed scheme, monotone or edge blocks are identified by examining the DCT coefficients of the block itself. In the first algorithm of the proposed scheme, a signal adaptive filter is applied to sub-image constructed by the DC components of DCT coded image to exploit the residual inter-block correlation between adjacent blocks. To further reduce artificial discontinuities due to blocking artifacts, the blocky image is re-divided into blocks in such a way that the corner of the original blocks comes at the center of new blocks. These discontinuities cause the high frequency components in the new blocks. In this paper, these high frequency components due to blocking artifacts in monotone area are eliminated using singular value decomposition (SVD) based filtering algorithm. It is well known that random noise is hard to compress whereas it is easy to compress the ordered information. Thus, lossy compression of noisy signal provides the required filtering of the signal.
\end{abstract}

Key words: Image compression, Image processing, Post-processing, Blocking artifacts, DCT, Singular value decomposition, Block transform coding.

\section{Introduction}

Objective of image compression is the reduction of storage and transmission cost. Many efficient coding techniques have been developed for image compression. Amongst them discrete cosine Transform (DCT) based compression scheme is used in many applications. At low and moderate compression ratios these schemes yields excellent reproduction without artifacts but at higher compression ratios it suffers from blocking artifacts. The reconstructed image from highly compressed data using discrete cosine transform (DCT) based algorithm has noticeable degradation due to blocking artifacts. This is the main drawback of the DCT based image compression algorithms and thus wavelet based image compression is used in new standard JPEG 2000. Many image compression standards (e.g., JPEG, MPEG, and H.263) are using DCT based compression schemes. The blocking artifacts in DCT based image compression cause grid noise in monotone area, staircase noise near the edges and corner outliers at the cross point of $\mathrm{M} \times \mathrm{M}$ block. Low pass filtering is inadequate to remove various types of noise present in different area of blocky image. Low pass filtering reduces grid noise in monotone area but it does not reduce the staircase noise near the edges.

Correspondence to: < vksrivastava12@rediffmail.com >

Recommended for acceptance by Umapada Pal and P. Nagabhusan

ELCVIA ISSN: 1577-5097

Published by Computer Vision Center / Universitat Autonoma de Barcelona, Barcelona, Spain 
Therefore, nonlinear space-variant or adaptive filter is required to reduce noise in the different regions of the image. Blocking artifacts are visible in the reconstructed image because the inter-block correlation is not taken into account during encoding and quantization process of DCT coefficients. At higher compression ratios, the blocking artifacts are more visible as very few coefficients are encoded.

Several post-processing algorithms to reduce blocking artifacts of block coded images have been proposed [1-14]. In nonlinear space-variant filtering [1], the image is divided into monotone area or edge area using edge information obtained from detecting edge by gradient operator. The approach given in [1] uses a two-dimensional (2D) low pass filter to reduce grid noise in monotone area and a directional onedimensional filter to reduce staircase noise along the edges. In adaptive filtering [2-7], the coefficients of 2D filter are chosen by edge information of the image. Weighted or un-weighted filtering is performed at the pixels in the monotone area or near the edge but no filtering is done at edge pixels. In [6], a 2D multiple notch filter is applied to reduce the blocking artifacts. Both nonlinear space-variant filtering and adaptive filtering require classification of image blocks, which is based on available edge information extracted from the received blocky image. Hence the performance of space-variant or adaptive filtering scheme degrades.

In [7-19], the blocking artifacts are reduced by processing the image in DCT domain itself. An approach for reducing the blocking artifact in frequency domain is presented in [9]. Mean squared difference of slope (MSDS) is used as a measure the blocking artifacts. It is shown in [9] that the expected value of the MSDS increases after quantizing the DCT coefficients. This approach removes the blocking effect by minimizing the MSDS, while imposing linear constraints corresponding to quantization bounds. Lakhani et al. [10] also used MSDS to reduce blocking effects. They have given a different solution for minimizing the MSDS. Triantafyllidis et al. [11] have proposed another method of minimizing MSDS, which involves diagonal neighboring pixels in addition to horizontal and vertical neighboring pixels. Then, for each block affected by blocking artifacts, DC and AC coefficients are recalculated for artifact reduction.

Jeon and Jeong [12] proposed a method for post-processing that gives minimum discontinuity of pixel values over block boundaries by compensating the loss of a coefficient's accuracy in the transform domain. In [13], Hsia et al. proposed transform-domain algorithm to effectively classify the characteristics of blocks and estimate the strength of the blocky effect. An adaptive finite impulse response (FIR) filter to effectively remove the blocky effect is also proposed. Chen et al. [14] introduced a DCT-domain postfiltering approach to reduce blocking artifacts, where the post-filter made use of the DCT coefficients of shifted blocks in order to obtain a close correlation between the DCT coefficients at the same frequency. The filtering adapts according to the local activity of each block to achieve simultaneous artifact reduction and detail preservation. However, the computational cost is rather high. In [15], Chang and Messerschmitt, proposed algorithms to manipulate compressed video in the transform domain. In [16], a method for efficiently assessing, and subsequently reducing, the severity of blocking artifacts in compressed image bit streams is proposed. The method operates only in the DCT domain. Hence, it is efficient as the signal need not be compressed or decompressed again. In the algorithm, blocking artifacts are modeled as 2-D step function. A fast DCT-domain algorithm extracts all parameters needed to detect and estimate the blocking artifacts, by exploiting several properties of the human vision system. Using the estimate of blockiness, a novel DCT-domain method is then developed which adaptively reduces the detected blocking artifacts. Luo and Ward [17] proposed a technique, which preserve the edge and texture information. This adaptive approach performs the blocking artifact reduction in both the DCT and spatial domains. For smooth regions, the continuity of original pixel levels in the same block and the correlation between the neighboring blocks is used to reduce the discontinuity of the pixels across the boundaries. For texture and edge regions, an edgepreserving smoothing filter is applied. Zhao et al. [18], proposed a DCT domain deblocking technique by shifting the blocks successively in horizontal and vertical directions, respectively, and simultaneously shrinking the undesired high frequency DCT coefficients.

Linear filtering is used to remove the additive noise but their major drawback is that they tend to blur the edges. Conventional techniques usually apply SVD to the whole image in a single, makes it computationally complex. By applying SVD sequentially into blocks of the image, the computational requirement significantly reduces. Non-linear filtering based on SVD method has been successfully used in past for various noise removal applications [20-23]. This technique does not require any additional a priori information. It is well known that random noise is hard to compress than the ordered information. Thus, 
lossy compression of noisy signal, with allowed loss set equal to the noise strength provides the required filtering of the signal.

In this paper, the proposed algorithms are described in Section 2. In first part of this section, the filtering of DC component by signal adaptive filter is discussed whereas in the second part the singular value decomposition (SVD) based block boundary discontinuity reduction algorithm is described. In Section 3 the results are presented. Finally, conclusions are given in Section 4.

\section{Proposed Scheme}

Blocking artifacts are mainly due to independent coding of different blocks. In original image a large inter-block correlation exits and some amount of it remains in the blocky decoded image. To exploit this, a signal adaptive filter is applied to a sub-image containing only the DC component of coded image. To further reduce the discontinuities, the blocky image is re-divided into blocks in such a way that the corner of the original blocks comes at the centre of new blocks. These discontinuities are reflected as AC component in the DCT of new blocks. A SVD-based filtering is used to reduce the discontinuities while preserving the edge details. These two algorithms will now be described in this section.

\subsection{Filtering of DC sub-image by Signal Adaptive Filter}

This algorithm is based on the assumption that the correlation among the DC coefficients of neighbouring block is high if all these blocks belong to monotone area. Here, a DC sub-image is constructed by taking only the DC coefficient of each block of the DCT coded image. A signal adaptive filter is applied to smooth the DC sub-image. Filtering mask of this filter is shown in Fig. 1. Initial value of the mask at centre (C) is taken as 0.75 whereas the value at other place (A, B, D, and E) is 0.0625 . Experimentally it is found that this value of filtering mask is giving good results.

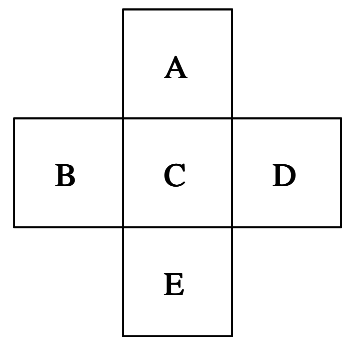

Fig.1: Filtering mask of Signal Adaptive Filter.

The image blocks are classified as the monotone block or the edge block simply by comparing the absolute value of sum of the AC coefficient of the block with a threshold. In this adaptive filtering scheme for the DC sub-image, if the centre $\mathrm{C}$ of the filtering mask is at DC component corresponding to the edge block then all surrounding coefficients A, B, D and $\mathrm{E}$ are made zero and this value remains unchanged. Since the blocking artifacts are more visible in monotone areas, it is assumed here that DC value correspond to monotone block is more correlated with the DC value of neighboring blocks. If the centre of mask is at DC component corresponding to the monotone block, then those values of filtering mask that are at the DC component corresponding to the edge block are decreased to zero whereas the weight of centre of mask is increased by the same amount. These filtered values are fed back in the original image blocks and inverse DCT is taken to reconstruct the image.

\subsection{SVD Based Algorithm for Block Boundary Discontinuities Reduction}

In the proposed algorithm Singular value decomposition (SVD) technique is used to reduce the discontinuities present in the DCT decompressed images. Followings are the steps in this algorithm:

Step 1. Division of blocky image into new blocks - The blocky image is re-divided into blocks in such a way that the corner of the original blocks comes at the centre of new blocks as shown in Fig. 2. This type of 
re-division captures maximum artificial discontinuities in the new blocks. These discontinuities are reflected as AC component in the DCT of the new blocks.

Step 2. Classification of blocks - To protect actual edges the classification of the new blocks into edge block and monotone block is required. Classification of block can be performed in DCT domain by examining the values of DCT coefficients. In this DCT based block classification scheme, the sum of absolute values of AC components is used to classify the blocks into the monotone block or the edge block. The average of sum of absolute values of AC components for all blocks is taken as the threshold for the classification.

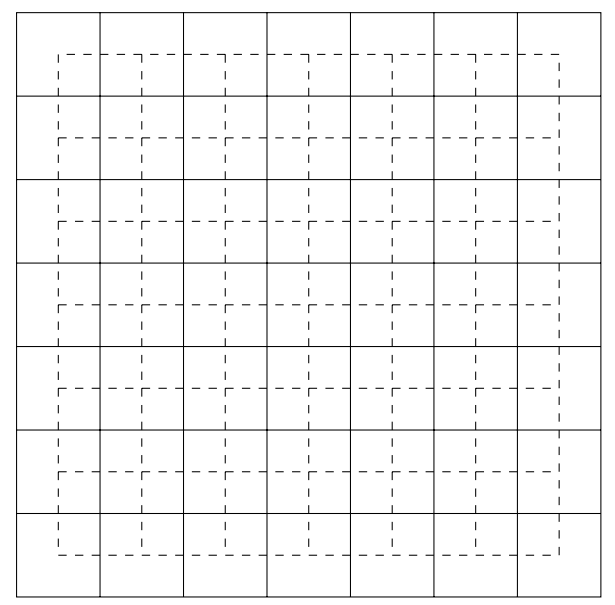

Fig.2: Original (solid line) and new position (dotted line) of blocks used for reducing discontinuities.

Step 3. Reduction of discontinuity - The approach of discontinuity reduction is based on the block-based, non-linear filtering algorithm. This filtering approach is based on the SVD algorithm. Linear filtering is used to remove the additive noise but their major drawback is that they tend to blur the edges. Conventional techniques usually apply SVD to the whole image, makes it computationally complex. In this paper a blockbased, non-linear filtering technique based on SVD is presented. By applying SVD sequentially to the blocks of the image, the computational requirement significantly reduces. Non-linear filtering based on SVD method has been successfully used in past for various noise removal applications [21-23]. This technique does not require any additional a priori information. It is well known that the random noise is hard to compress whereas the ordered information can be compressed easily. Thus, lossy compression of noisy signal, with allowed loss set equal to the noise strength provides the required filtering of the signal. Proposed SVD-based filtering can effectively reduce the discontinuities while preserving the edge details. The SVD algorithm can be viewed as a lossy compression scheme. For the monotone blocks, the discontinuities are hard to compress as compared to smooth area therefore SVD algorithm allows to filter out these discontinuities. Derivation of the optimum threshold to distinguish significant and insignificant singular values for reducing discontinuity is still a problem with this approach, which must be investigated. The threshold calculation requires the additional signal strength due to discontinuities. The theory of SVD-based filtering is discussed below.

Any $m \times n$ real-valued matrix $A$ can be decomposed uniquely as

$$
A=U \Sigma V^{T}=\sum_{i=0}^{k} \alpha_{i} \boldsymbol{u}_{i} \boldsymbol{v}_{i}^{T}
$$

where $U$ and $V$ are the orthogonal matrices with column vectors $u_{i}$ and $v_{i}$ and $\sum=\operatorname{diag}\left(\alpha_{1}, \alpha_{2}, \alpha_{3}, \ldots \alpha_{n}\right)$ is a diagonal matrix. The diagonal elements of $\Sigma$ can be arranged in descending order and are called the singular values of $A$. Thus, SVD provides the rank $r$ of a matrix, which is equal to the number of non-zero singular values of the matrix. If rank of the matrix $A$ is $r$ then $\alpha_{1} \geq \alpha_{2} \geq \ldots \alpha_{r}>\alpha_{r+1}=\ldots=\alpha_{n}=0$. 
For additive noise model, observation matrix $B$ is equal to $A+E$, where $E$ is a random noise matrix of full rank. So, the last $(n-r)$ singular values of $B$ are small but not necessarily zero.

Let $B$ be the $M \times N$ image divided into $k \times k$ image blocks $B_{k}$. Each block can be expressed as the weighted sum of basis images and weights are the singular values. Setting "non-significant" singular values to zero results in lossy compression. The amount of loss is controlled by a threshold value $\varepsilon$. The effectiveness of algorithm depends upon the value of $\varepsilon$. If $\varepsilon$ is very small then most of the noise remains but if $\varepsilon$ is very large then image information is lost. In the proposed algorithm, $5 \%$ of the largest singular value is taken as the threshold value $\varepsilon$. Thus, the main steps of SVD-based filtering algorithm are-

1. Divide $M \times N$ image $B$ into $k \times k$ blocks $B_{k}$.

2. Apply SVD to each block and set the singular values less than a threshold $\varepsilon$ to zero.

$$
B_{k}=U \Sigma V^{T}=\sum_{i=0}^{k} \alpha_{i} \boldsymbol{u}_{i} \boldsymbol{v}_{i}^{T}
$$

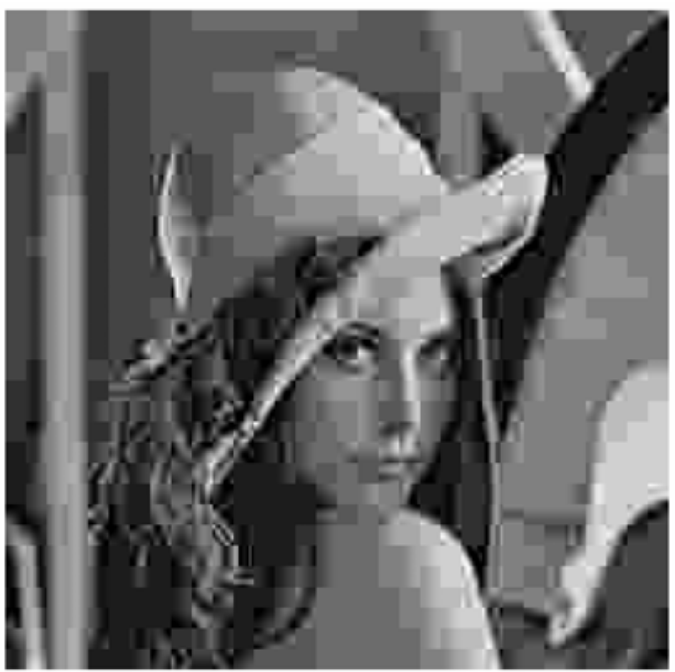

(a)

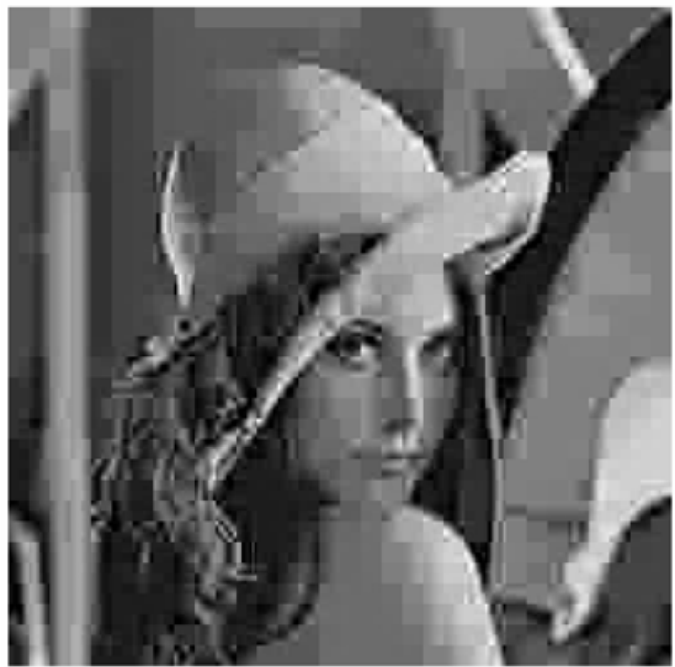

(c)

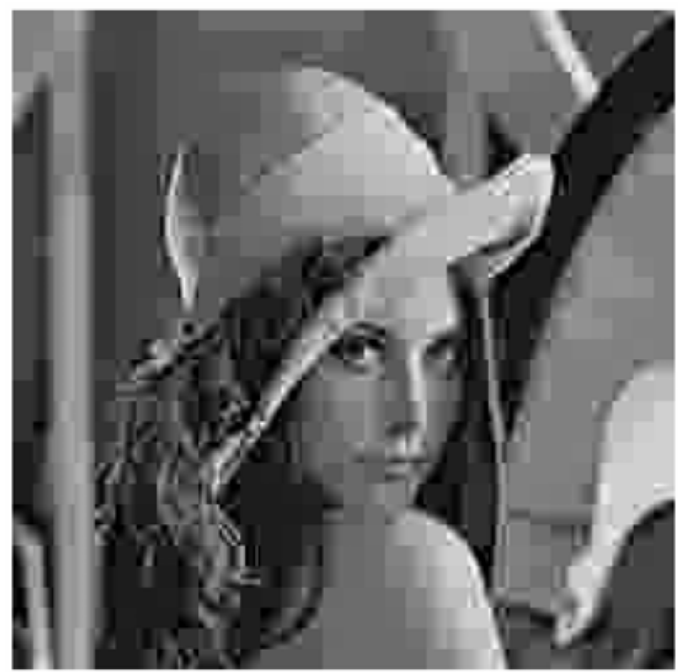

(b)

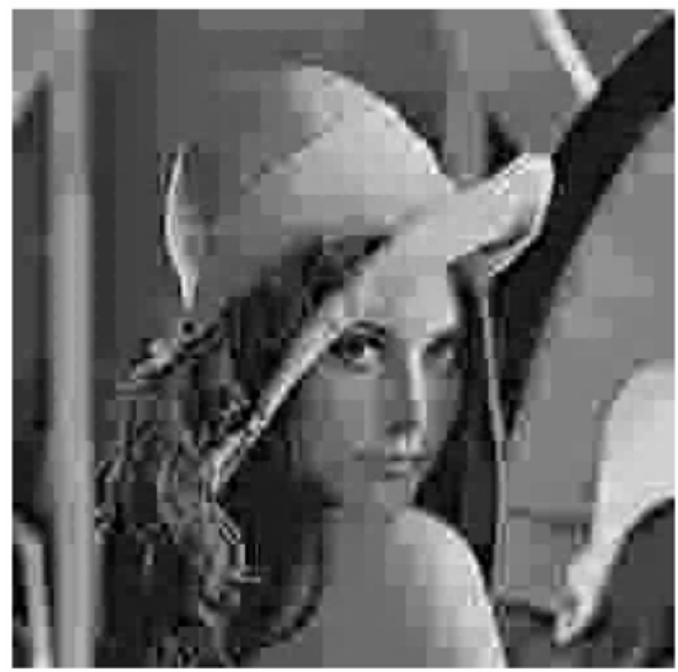

(d)

Fig.4: Post-processed Lenna image compressed at 0.234 bits/pixel by various algorithms. (a) DCT decoded. (b) DC component filtering. (c) Proposed SVD based algorithm (d) With corner outliers reduction. 


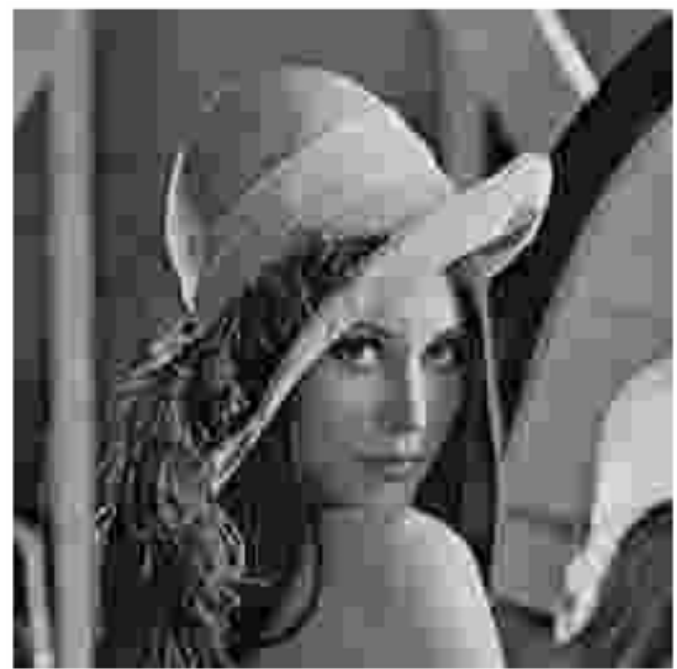

(a)

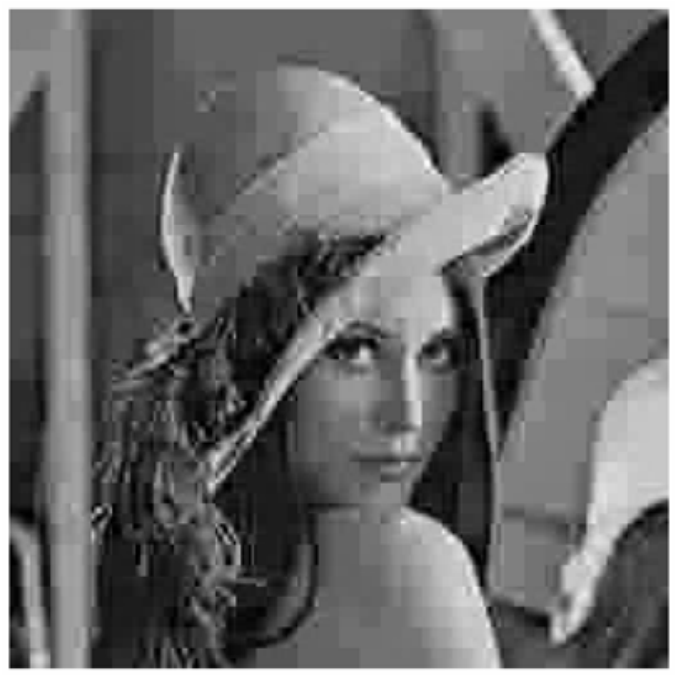

(c)

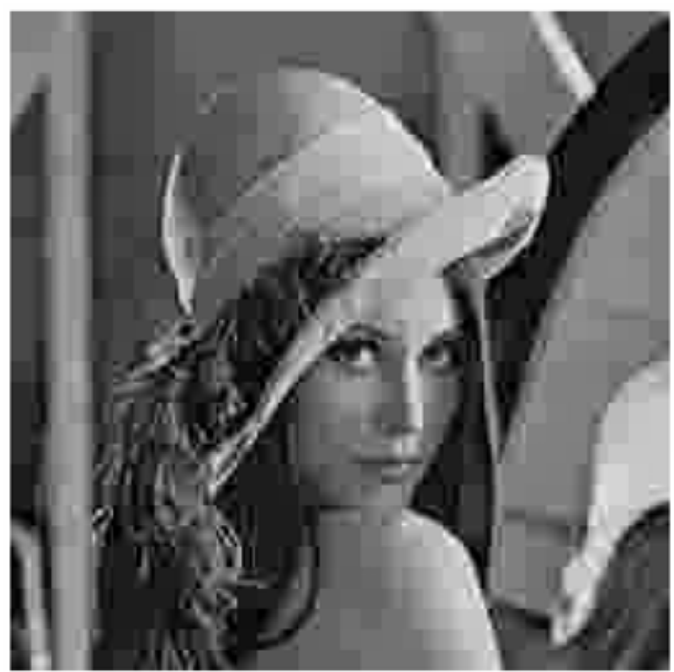

(b)

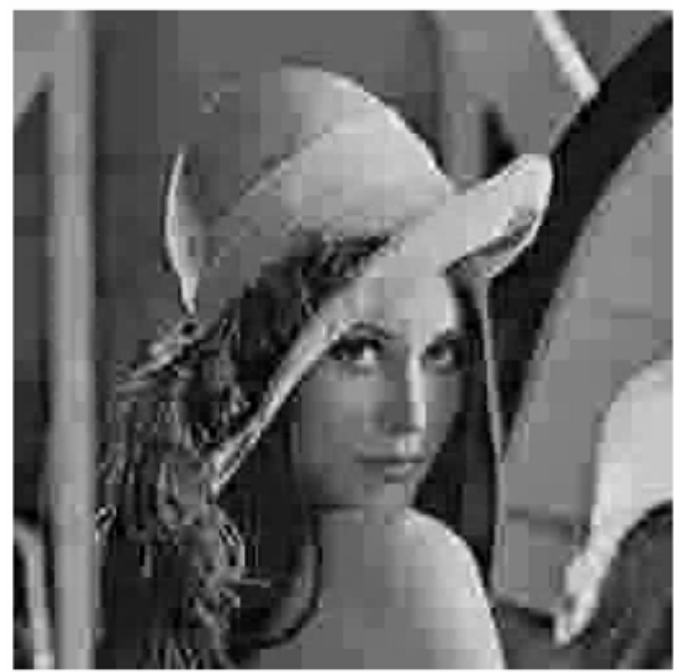

(d)

Fig.5: Post-processed Lenna image compressed at 0.281 bits/pixel by various algorithms. (a) DCT decoded. (b) DC component filtering. (c) Proposed SVD based algorithm (d) With corner outliers reduction.

Let $r$ be the number of non-zero singular values greater than a threshold $\varepsilon$. If $\Sigma^{\prime}$ is resultant diagonal matrix of singular value after threshold then the reconstructed block will be

$$
B_{k}^{\prime}=U \Sigma^{\prime} V^{T}=\sum_{i=0}^{r} \alpha_{i} u_{i} v_{i}^{T}
$$

3. Replace $B_{k}$ with $B_{k}{ }^{\prime}$ where $B_{k}{ }^{\prime}$ is resultant block after setting the singular values less than a threshold $\varepsilon$ to zero.

4. Apply step (2) and (3) to all the blocks and reconstruct the image.

Note that this algorithm is applied to the new blocks obtained by re-division of the blocky image into blocks as discussed in step 1 of discontinuity reduction algorithm. It is found that the reduction in blocking artifacts cannot be achieved with the original blocks. This approach reduces the blocking artifacts more effectively but its computational complexity is more because SVD algorithm has to be applied to each of the block. 


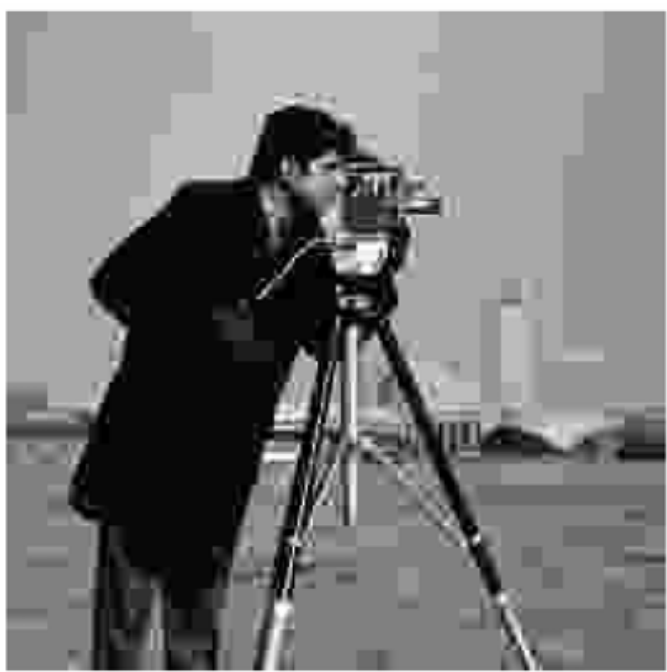

(a)

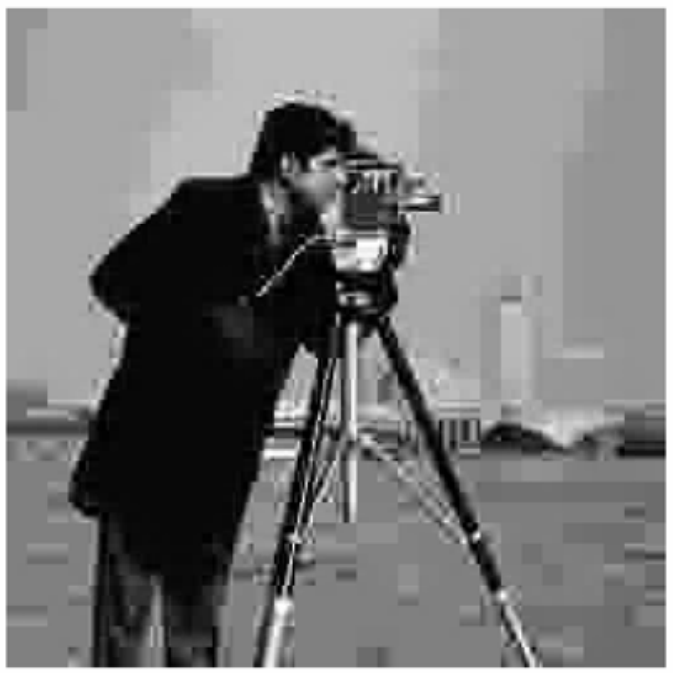

(c)

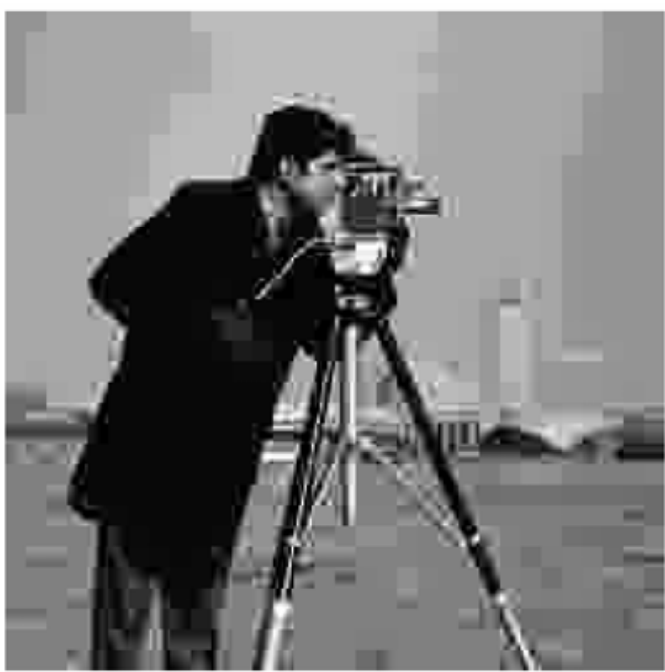

(b)

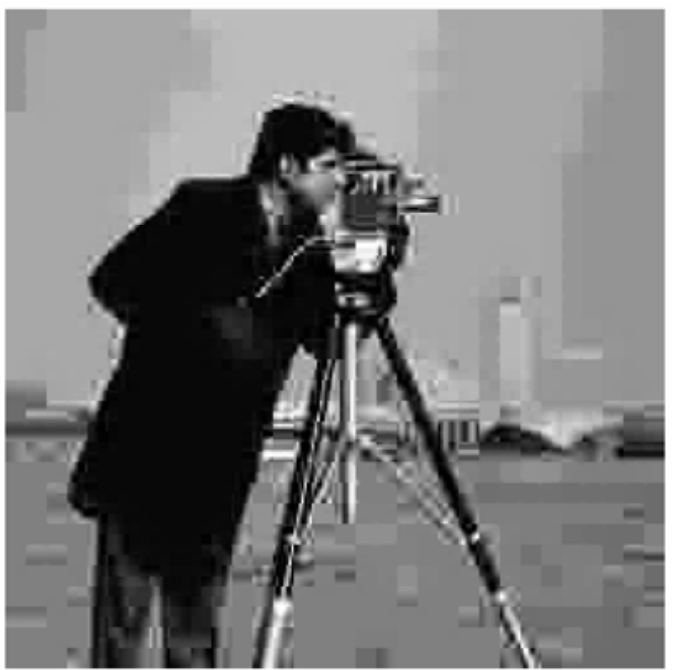

(d)

Fig.6: Post-processed Cameraman image compressed at 0.239 bits/pixel by various algorithms. (a) DCT decoded. (b) DC component filtering. (c) Proposed SVD based algorithm (d) With corner outliers reduction.

\subsection{Corner Outliers Detection and Replacement}

As discussed in [4], corner outliers are visible at the cross point of $M \times M$ block when the corner pixel of one block is very large or very small as compared to corner pixels of the neighbouring blocks (See Fig. 3).

\begin{tabular}{|l|l|l|l|}
\hline$A_{3}$ & $A_{2}$ & $B_{2}$ & $B_{3}$ \\
\hline$A_{1}$ & $A$ & $B$ & $B_{1}$ \\
\hline$C_{1}$ & $C$ & $D$ & $D_{1}$ \\
\hline$C_{3}$ & $C_{2}$ & $D_{2}$ & $D_{3}$ \\
\hline
\end{tabular}

Fig. 3: Pixels used in corner outliers detection and replacement algorithm. 


\begin{tabular}{|c|c|c|c|c|c|c|c|c|c|c|}
\hline \multirow[t]{2}{*}{ Image } & \multirow{2}{*}{ 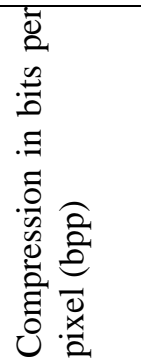 } & \multirow{2}{*}{ 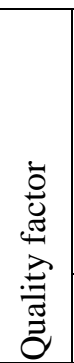 } & \multicolumn{2}{|c|}{ DCT Decoded } & \multicolumn{2}{|c|}{$\begin{array}{l}\text { DC Component } \\
\text { Filtering }\end{array}$} & \multicolumn{2}{|c|}{$\begin{array}{l}\text { Post-processed } \\
\text { additionally by } \\
\text { SVD based } \\
\text { Block Boundary } \\
\text { Discontinuities } \\
\text { Reduction }\end{array}$} & \multicolumn{2}{|c|}{$\begin{array}{l}\text { Further processed } \\
\text { by Corner } \\
\text { Outliers } \\
\text { Reduction }\end{array}$} \\
\hline & & & PSNR & BPSNR & PSNR & BPSNR & PSNR & BPSNR & PSNR & BPSNR \\
\hline \multirow{4}{*}{ Lena } & 0.23421 & 5 & 25.109 & 24.381 & 25.207 & 24.506 & 25.244 & 24.624 & 25.376 & 24.889 \\
\hline & 0.28123 & 7 & 26.379 & 25.809 & 26.433 & 25.870 & 26.427 & 25.978 & 26.526 & 26.184 \\
\hline & 0.32252 & 9 & 27.338 & 26.775 & 27.316 & 26.778 & 27.291 & 26.889 & 27.372 & 27.061 \\
\hline & 0.36054 & 11 & 28.061 & 27.591 & 28.021 & 27.585 & 27.950 & 27.703 & 28.004 & 27.819 \\
\hline \multirow{4}{*}{$\begin{array}{l}\text { Camera } \\
\text { man }\end{array}$} & 0.23850 & 5 & 24.446 & 24.032 & 24.539 & 24.135 & 24.556 & 24.207 & 24.621 & 24.347 \\
\hline & 0.27774 & 7 & 25.523 & 25.197 & 25.582 & 25.263 & 25.591 & 25.344 & 25.672 & 25.519 \\
\hline & 0.31760 & 9 & 26.189 & 25.972 & 26.234 & 26.023 & 26.260 & 26.111 & 26.324 & 26.254 \\
\hline & 0.35524 & 11 & 26.765 & 26.670 & 26.814 & 26.721 & 26.846 & 26.845 & 26.878 & 26.917 \\
\hline \multirow{4}{*}{ Bird } & 0.25709 & 5 & 28.779 & 27.991 & 29.007 & 28.265 & 29.040 & 28.382 & 29.193 & 28.687 \\
\hline & 0.28622 & 7 & 30.274 & 29.582 & 30.467 & 29.802 & 30.494 & 29.952 & 30.603 & 30.175 \\
\hline & 0.31366 & 9 & 31.562 & 30.731 & 31.768 & 30.932 & 31.803 & 31.147 & 31.883 & 31.307 \\
\hline & 0.33841 & 11 & 32.558 & 31.811 & 32.714 & 31.962 & 32.714 & 32.237 & 32.757 & 32.325 \\
\hline \multirow{4}{*}{ Goldhill } & 0.21820 & 5 & 24.309 & 23.817 & 24.412 & 23.973 & 24.422 & 24.018 & 24.523 & 24.231 \\
\hline & 0.26752 & 7 & 25.381 & 24.984 & 25.411 & 25.038 & 25.426 & 25.116 & 25.508 & 25.294 \\
\hline & 0.31696 & 9 & 26.076 & 25.756 & 26.081 & 25.784 & 26.100 & 25.881 & 26.151 & 25.992 \\
\hline & 0.36511 & 11 & 26.638 & 26.410 & 26.638 & 26.420 & 26.657 & 26.507 & 26.689 & 26.579 \\
\hline \multirow{4}{*}{ Tulips } & 0.30329 & 5 & 23.126 & 22.407 & 23.118 & 22.436 & 23.109 & 22.485 & 23.236 & 22.739 \\
\hline & 0.37182 & 7 & 24.294 & 23.781 & 24.240 & 23.758 & 24.229 & 23.832 & 24.314 & 24.012 \\
\hline & 0.43853 & 9 & 25.055 & 24.597 & 24.963 & 24.545 & 24.943 & 24.626 & 24.994 & 24.734 \\
\hline & 0.49804 & 11 & 25.640 & 25.256 & 25.545 & 25.193 & 25.522 & 25.288 & 25.544 & 25.336 \\
\hline \multirow{4}{*}{ Bridge } & 0.23756 & 5 & 22.697 & 22.266 & 22.744 & 22.338 & 22.756 & 22.397 & 22.836 & 22.566 \\
\hline & 0.30291 & 7 & 23.709 & 23.358 & 23.718 & 23.374 & 23.732 & 23.448 & 23.795 & 23.583 \\
\hline & 0.37014 & 9 & 24.420 & 24.144 & 24.410 & 24.140 & 24.418 & 24.211 & 24.460 & 24.303 \\
\hline & 0.42838 & 11 & 24.973 & 24.793 & 24.958 & 24.791 & 24.945 & 24.877 & 24.971 & 24.936 \\
\hline \multirow{4}{*}{ Einstein } & 0.22810 & 5 & 25.647 & 25.431 & 25.769 & 25.605 & 25.790 & 25.648 & 25.875 & 25.839 \\
\hline & 0.27032 & 7 & 27.048 & 26.756 & 27.106 & 26.845 & 27.115 & 26.892 & 27.180 & 27.035 \\
\hline & 0.30938 & 9 & 27.948 & 27.660 & 27.977 & 27.713 & 27.986 & 27.769 & 28.038 & 27.882 \\
\hline & 0.34855 & 11 & 28.762 & 28.598 & 28.786 & 28.647 & 28.803 & 28.713 & 28.829 & 28.772 \\
\hline \multirow{4}{*}{ House } & 0.26597 & 5 & 23.032 & 22.678 & 23.078 & 22.748 & 23.095 & 22.800 & 23.170 & 22.962 \\
\hline & 0.32658 & 7 & 24.091 & 23.810 & 24.105 & 23.834 & 24.114 & 23.891 & 24.174 & 24.022 \\
\hline & 0.38207 & 9 & 24.809 & 24.680 & 24.807 & 24.692 & 24.825 & 24.764 & 24.865 & 24.854 \\
\hline & 0.43426 & 11 & 25.373 & 25.277 & 25.370 & 25.285 & 25.382 & 25.359 & 25.407 & 25.415 \\
\hline \multirow{4}{*}{ Rice } & 0.39456 & 5 & 24.834 & 23.979 & 24.812 & 24.040 & 24.802 & 24.089 & 24.983 & 24.446 \\
\hline & 0.47311 & 7 & 25.985 & 25.256 & 25.991 & 25.275 & 26.003 & 25.341 & 26.125 & 25.583 \\
\hline & 0.54449 & 9 & 26.859 & 26.239 & 26.871 & 26.255 & 26.887 & 26.342 & 26.981 & 26.534 \\
\hline & 0.61228 & 11 & 27.480 & 26.971 & 27.492 & 26.985 & 27.479 & 27.040 & 27.534 & 27.155 \\
\hline
\end{tabular}

Table 1: Performance of the proposed algorithms on various compressed images: Comparison of PSNR and BPSNR (in $\mathrm{dB}$ ) obtained by various algorithms 


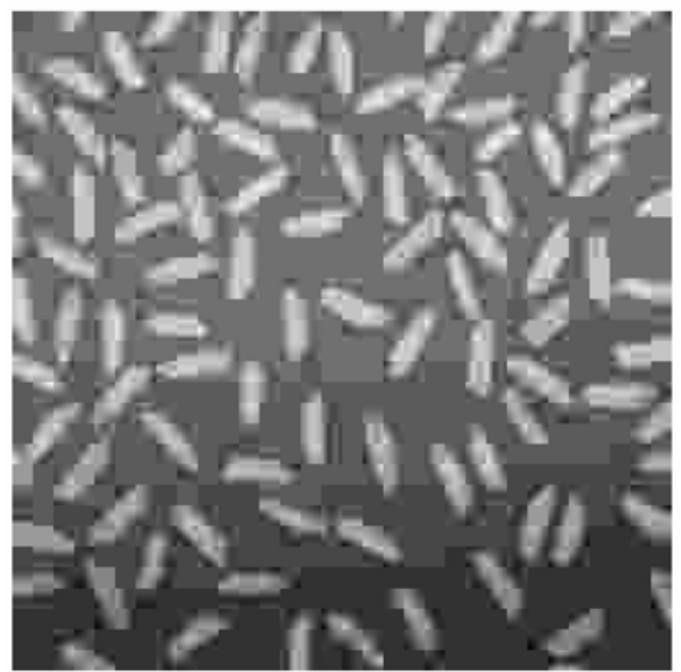

(a)

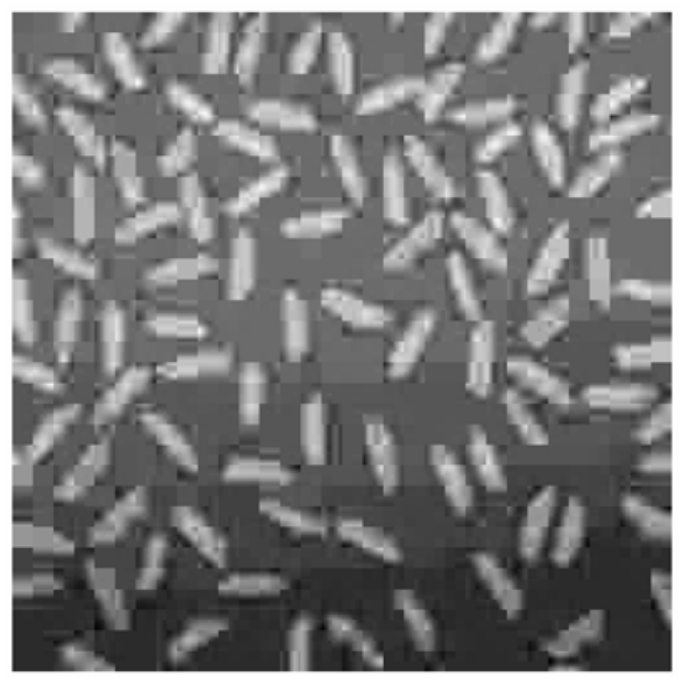

(c)

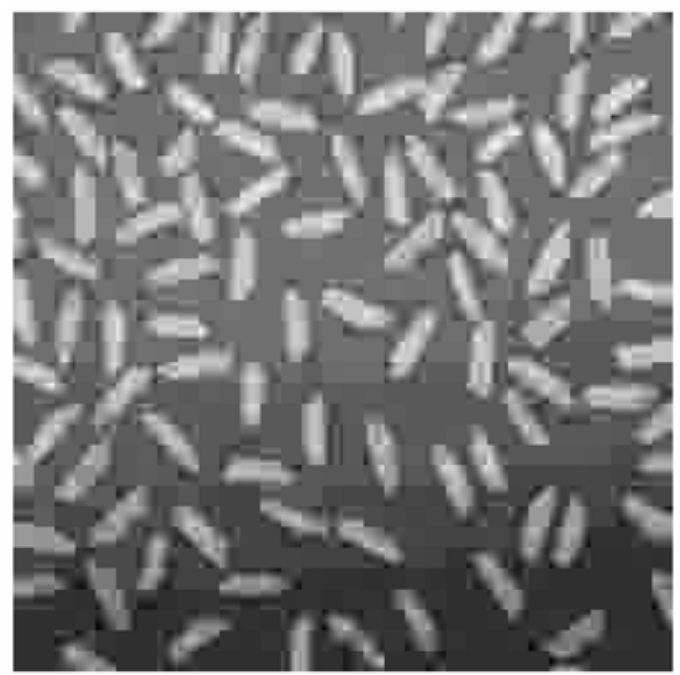

(b)

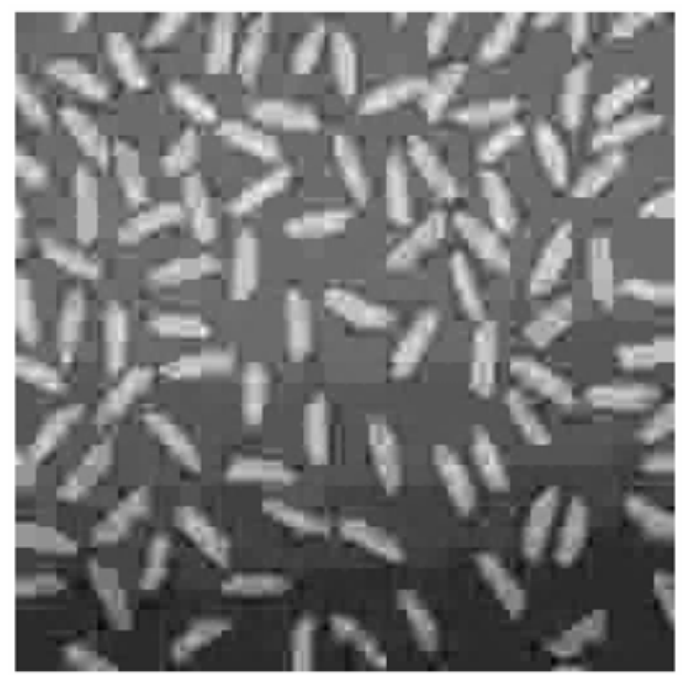

(d)

Fig.7: Post-processed Rice image compressed at 0.395 bits/pixel by various algorithms. (a) DCT decoded. (b) DC component filtering. (c) Proposed SVD based algorithm (d) With corner outliers reduction.

The corner outliers detection and replacement algorithm [4] is used here to further improve the processed image obtained from the proposed algorithm. In this algorithm, each corner pixel is compared with its neighbours to detect the corner outliers whereas to reduce this effect the corner $A$ and its neighbours $\left(A_{l}\right.$ and $A_{2}$ ) are replaced by its weighted average as given by:

$$
\begin{aligned}
& A^{\prime}=(4 A+B+C+2 D+4) / 8 \\
& A_{1}^{\prime}=\left(A^{\prime}+3 A_{1}+2\right) / 4 \\
& A_{2}^{\prime}=\left(A^{\prime}+3 A_{2}+2\right) / 4
\end{aligned}
$$




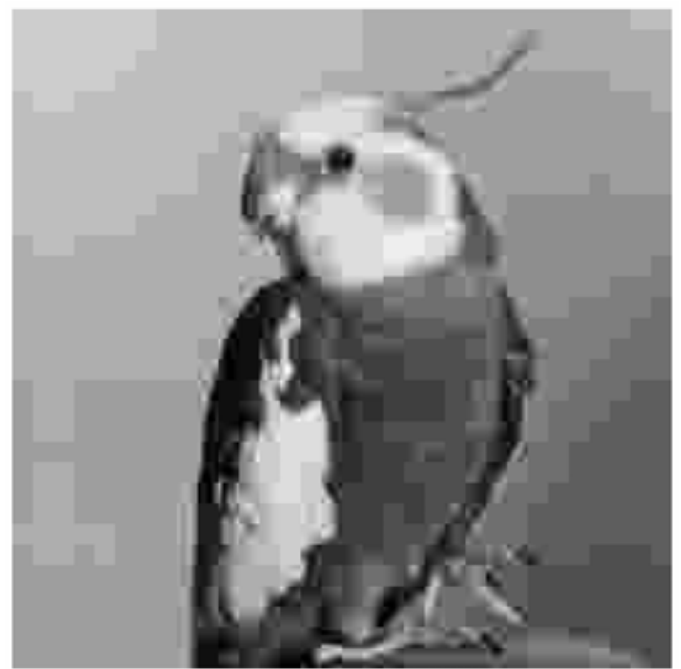

(a)

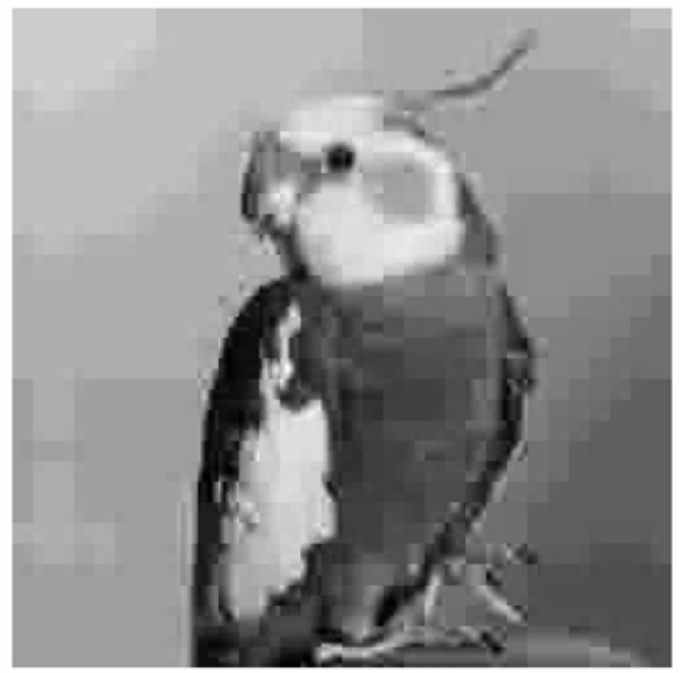

(c)

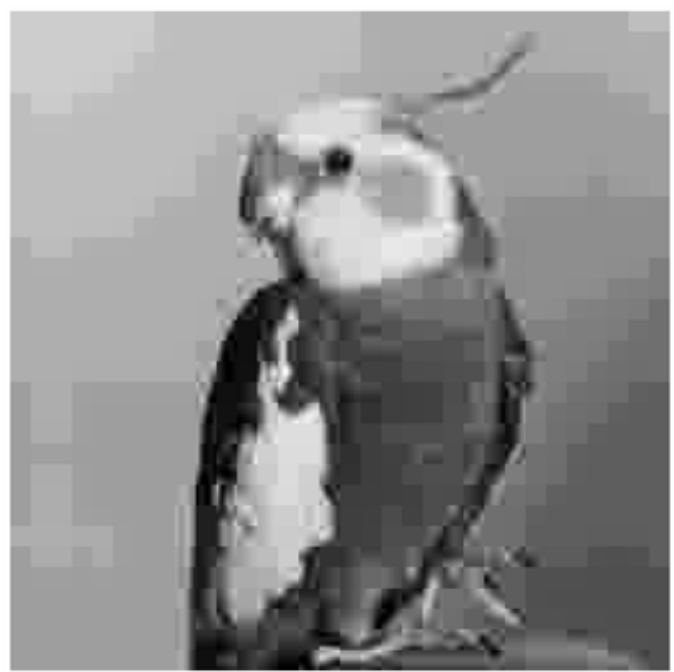

(b)

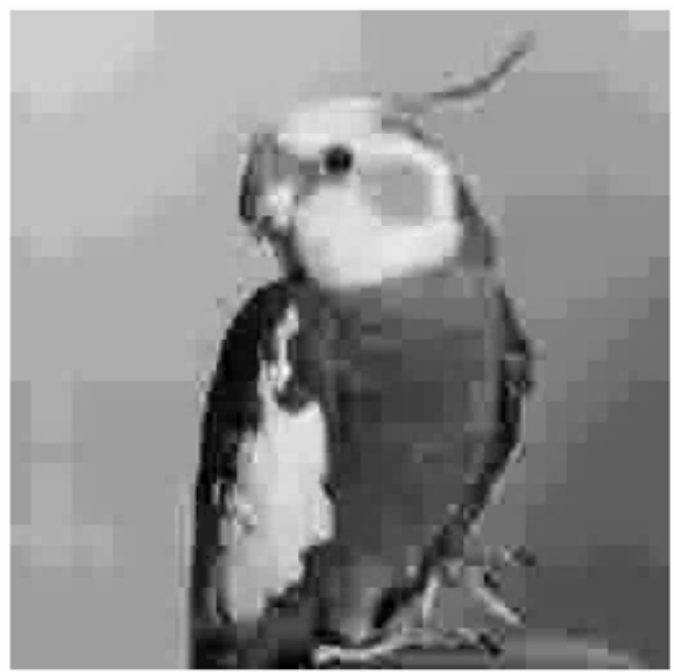

(d)

Fig.8: Post-processed Bird image compressed at 0.286 bits/pixel by various algorithms. (a) DCT decoded. (b) DC component filtering. (c) Proposed SVD based algorithm (d) With corner outliers reduction.

\section{Results}

In this section, results of the algorithms applied on several images are presented. The algorithm is implemented with MATLAB. To get blocky image at different compression ratio, a baseline JPEG like algorithm is implemented and then this blocky image is post-processed by this algorithm. Various results are given in Table 1. Various quality measures are calculated to see the performances of these algorithms. The results of the algorithms are compared with baseline JPEG like DCT decoded image. Peak signal to noise ratio (PSNR) is defined as:

$$
P S N R \text { in } d B=10 \log _{10}\left(\frac{255^{2}}{M S E}\right)
$$

where 255 is the peak signal for 8 bit PCM. MSE is mean square error given by:

$$
M S E=\frac{1}{N^{2}}\left[\sum_{i=0}^{N-1} \sum_{j=0}^{N-1}\left(f_{i j}-\tilde{f}_{i j}\right)^{2}\right]
$$




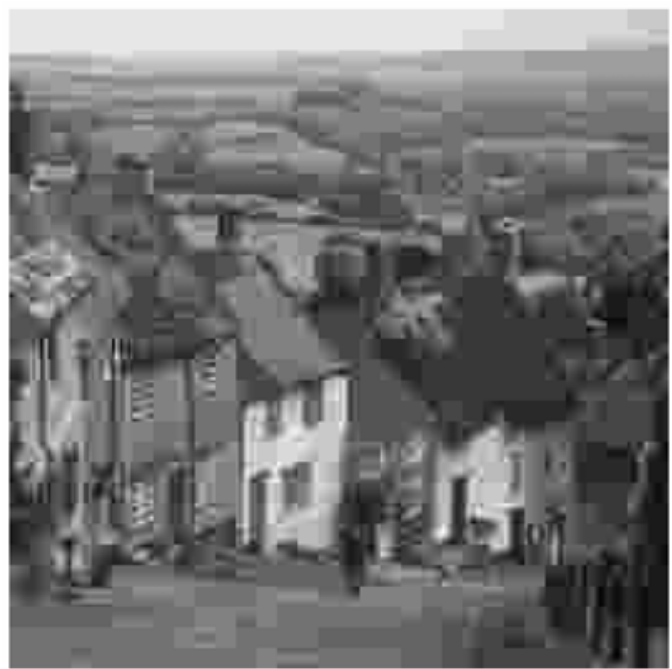

(a)

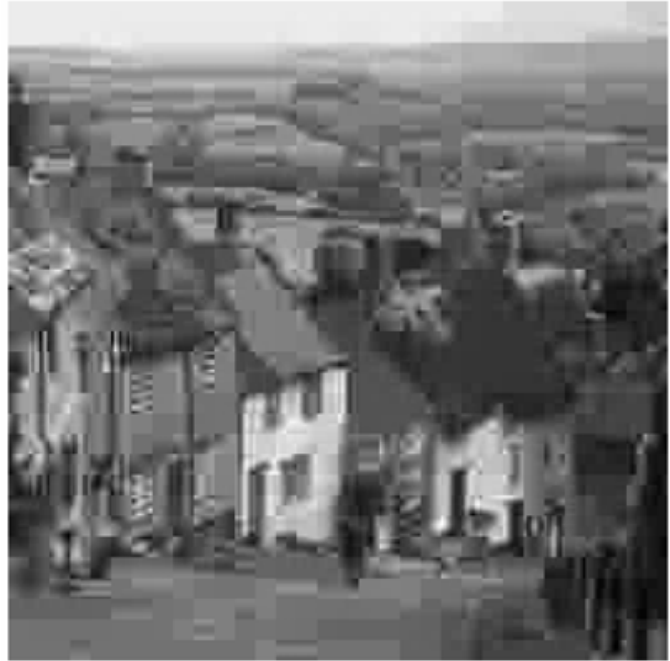

(c)

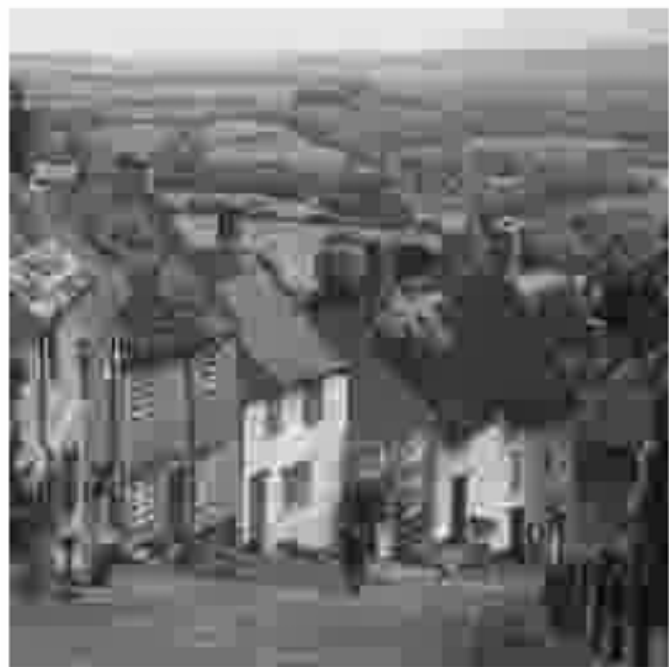

(b)

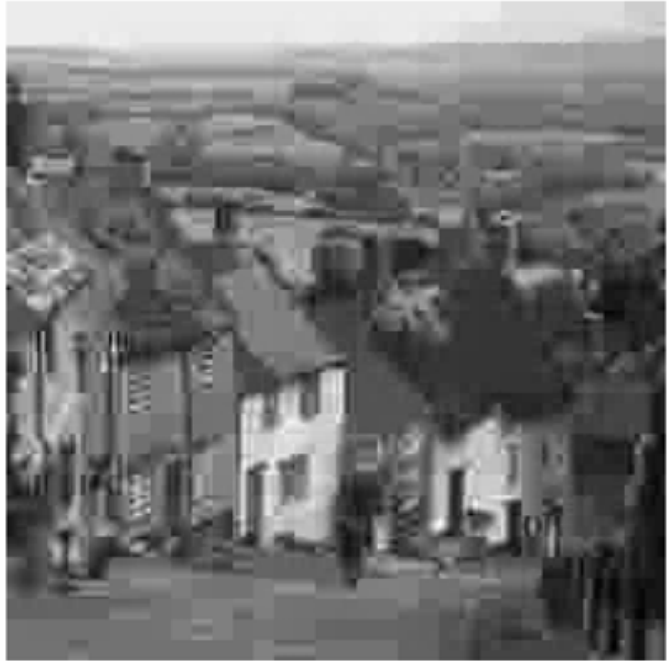

(d)

Fig.9: Post-processed Goldhill image compressed at 0.268 bits/pixel by various algorithms. (a) DCT decoded. (b) DC component filtering. (c) Proposed SVD based algorithm (d) With corner outliers reduction.

where $\boldsymbol{f}_{i j}$ and $\tilde{f}_{i j}$ are the pixel values at position $(i, j)$ of original and decoded image respectively.

Subjective quality of an image depends on the properties of human visual system (HVS), as the discontinuities are more visible in monotone or slowly varying areas. Therefore, the PSNR is only the rough indicator of image quality and does not reflect the true measure of blocking artifacts. A new discontinuity measure is defined. The block-boundary PSNR (BPSNR) is defined in the same way as PSNR but only one pixel from both side of block boundary are considered for the calculation of MSE.

\subsection{Comparison of the Performances of Various Algorithms Applied on Various Images}

Filtering of the sub-image consisting of DC components by signal adaptive filter reduces blocking artifacts with very low computational cost whereas the SVD based algorithm for block boundary discontinuities reduction shows significant reduction in blocking artifacts. Finally corner outliers detection and replacement algorithm is applied to further improve the performance. The results are presented in figures 4-11 and Table 1. Various terms used in the results and comparison of the performances of different 


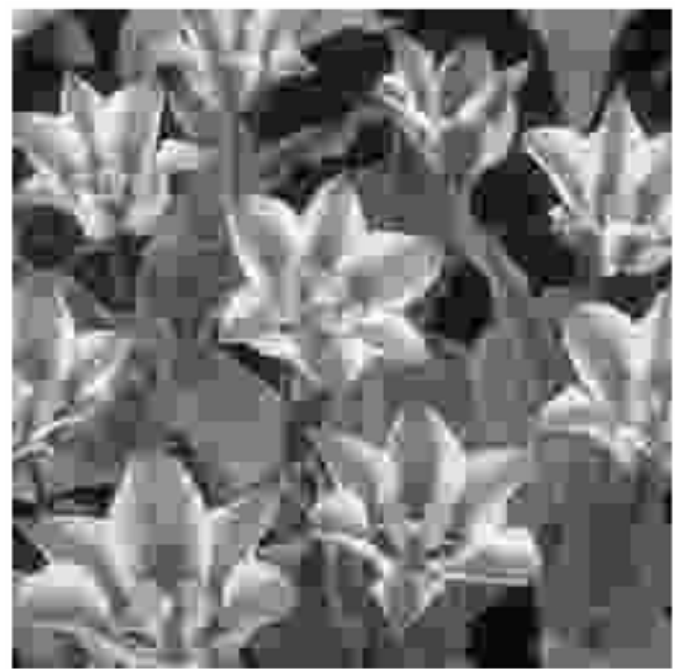

(a)

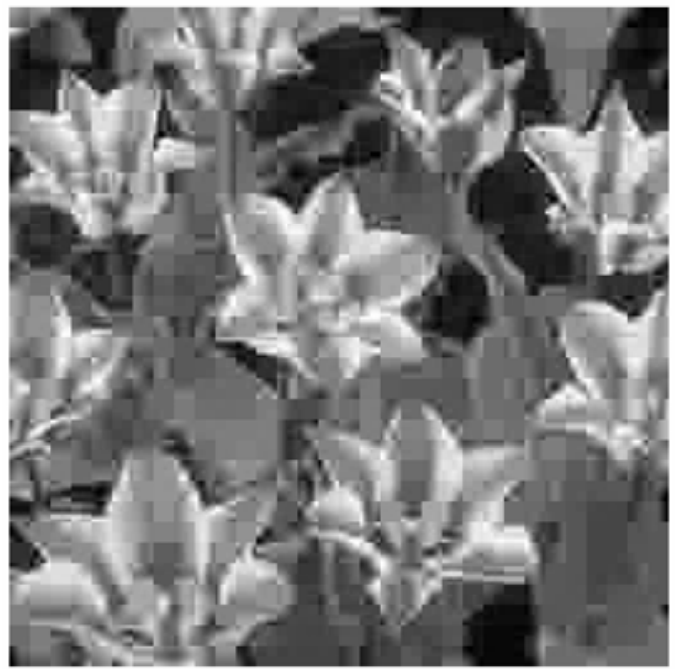

(c)

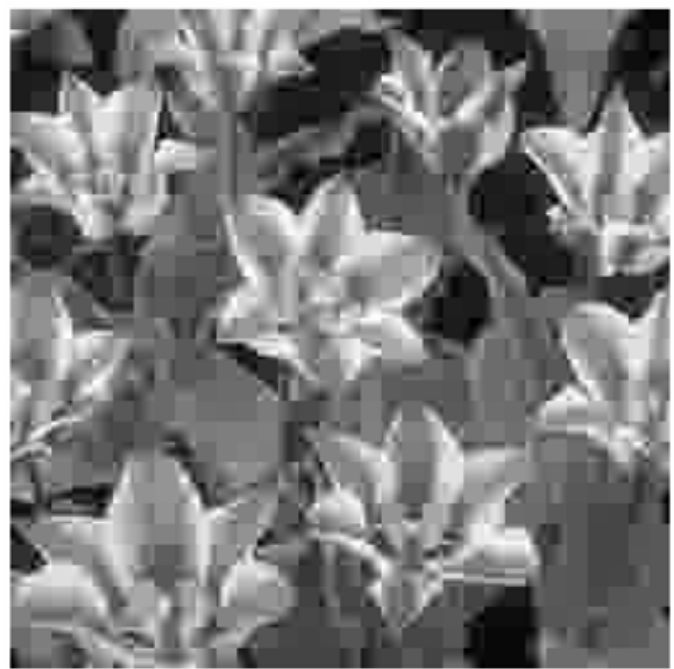

(b)

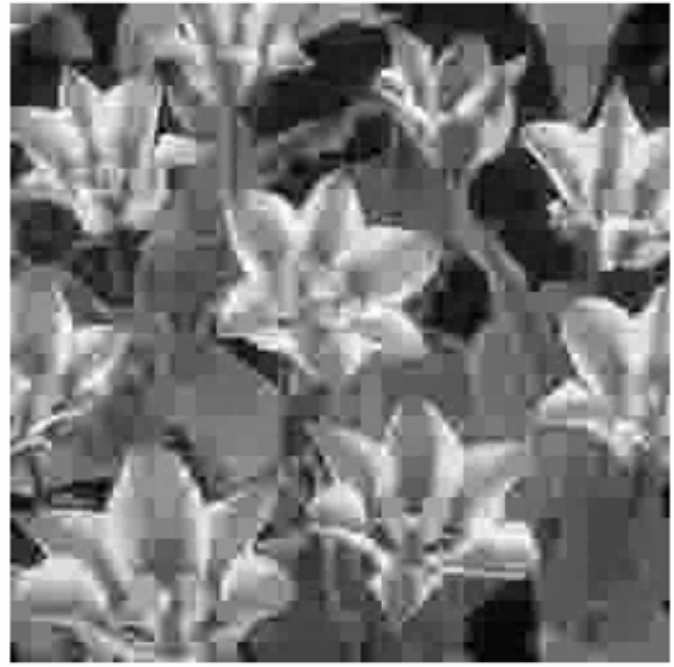

(d)

Fig.10: Post-processed Tulip image compressed at 0.303 bits/pixel by various algorithms. (a) DCT decoded. (b) DC component filtering. (c) Proposed SVD based algorithm (d) With corner outliers reduction.

algorithms will now be discussed. The image is first compressed for different compression ratios by using baseline JPEG like Algorithm. Then it is decompressed and named as the DCT decoded image. The first algorithm, i. e., the DC component filtering is applied to the DCT compressed image to smooth the DC components. This scheme shows significant reduction in blocking artifacts without much computation complexity. This can be observed from the figures 4-11. It can also be verified by calculating PSNR and block-boundary PSNR. There is slight improvement in overall PSNR whereas the improvement in the blockboundary PSNR (BPSNR) is more. These results are given in Table 1.

On this resultant image proposed algorithm, namely, the SVD based block boundary discontinuities reduction is applied to further reduce the blocking artifacts. The performance of this algorithm improves as the compression ratio increases. The reduction of discontinuity signifies (see Table 1) blocking artifacts are reduced which can also be observed visually in figures 4-11. It can also be seen from Table 1 that the improvement in PSNR for block boundary pixels is more as compared to over all PSNR. Finally corner outliers detection and replacement algorithm (i. e. corner outliers reduction algorithm) is also applied to post processed image. Thus proposed method for blocking-artifact reduction exhibits satisfactory performance as compared to other post-processing techniques. 


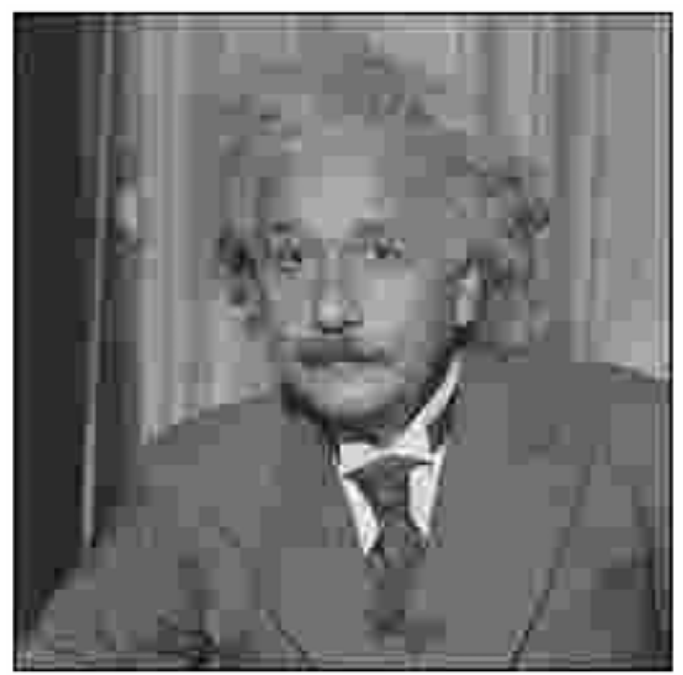

(a)

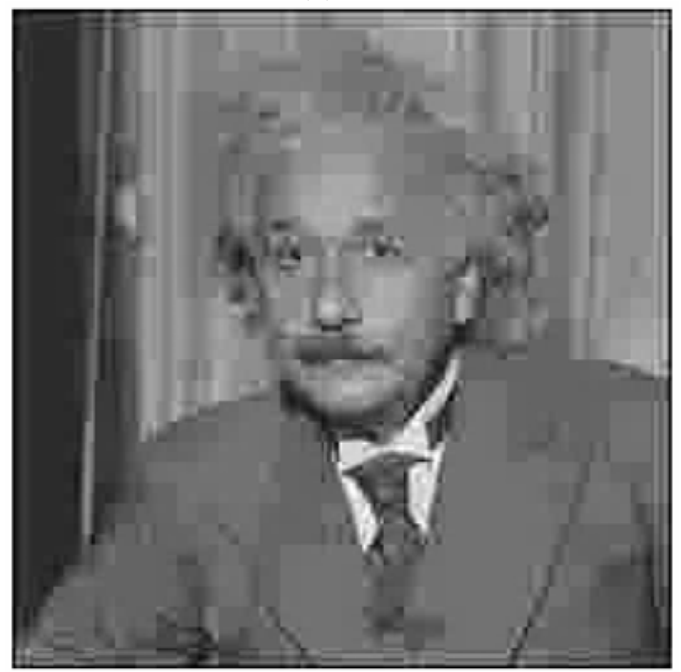

(c)

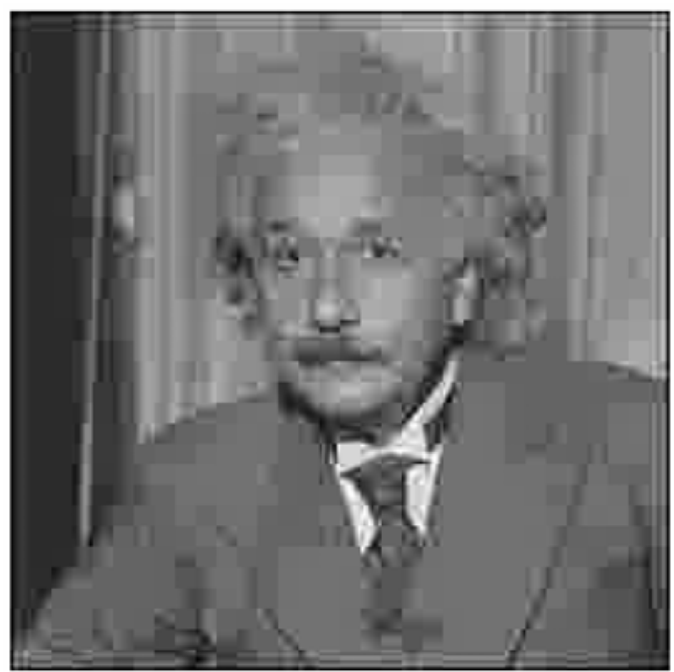

(b)

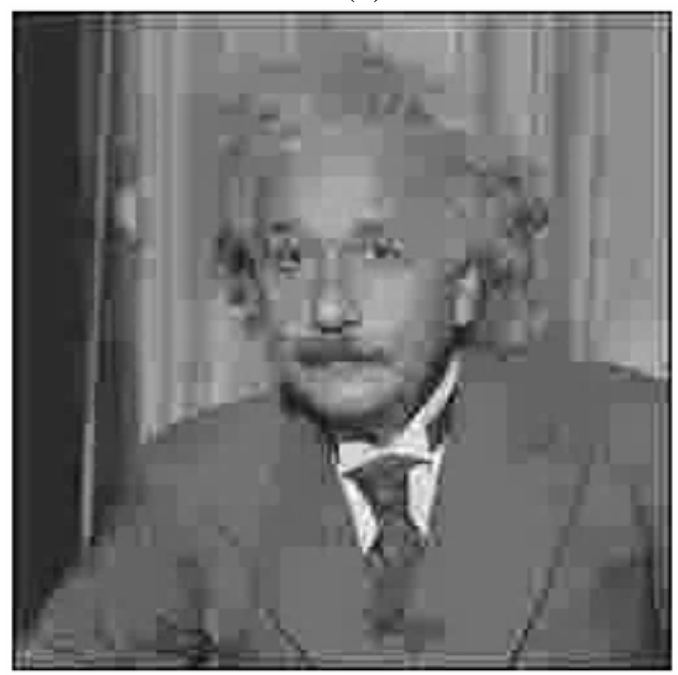

(d)

Fig.11: Post-processed Einstein image compressed at 0.27 bits/pixel by various algorithms. (a) DCT decoded. (b) DC component filtering. (c) Proposed SVD based algorithm (d) With corner outliers reduction.

\section{Conclusions}

In this paper, two effective algorithms for reducing blocking artifacts from the DCT coded image are proposed. The main feature of these algorithms is that it can preserve the high frequency components while smoothing out the blocking artifacts. In first algorithm the signal adaptive filter is applied only to a subimage consisting of DC components of the DCT coded image. This algorithm reduces the blocking artifacts with very low computational cost. In the second algorithm proposed in this paper, the discontinuity reduction is based on SVD-based filtering scheme. It reduces the blocking artifacts effectively but its computational complexity is slightly more because SVD algorithm has to be applied to each of the block. These results show that the proposed algorithms significantly reduce the blocking artifacts of DCT coded images as judged by both objective and subjective image qualities.

\section{References}

[1] B. Ramamurthi, A. Gersho, Nonlinear space-variant post-processing of block coded images, IEEE Trans. Accoust. Speech Signal Process. ASSP-34(5): 1258-1268, 1986. 
[2] C.J. Kuo and R.J. Hsieh, Adaptive post-processor for block encoded images, IEEE Trans. Circuit Syst. Video Technol., 5 (4): 298-304, August 1995.

[3] Y.L. Lee, H.C. Kim, and H.W. Park, Blocking effect reduction of JPEG images by signal adaptive filtering, IEEE Trans. on Image Processing, 7 (2): 229-234, February 1998.

[4] H. W. Park and Y. L. Lee, A post-processing method for reducing quantization effects in low bit-rate moving picture coding, IEEE Trans. Circuit Syst. Video Technol., 9(1): 161-171, February 1999.

[5] S.D. Kim, J. Yi, H.M. Kim, and J.B. Ra, A deblocking filter with two separate modes in block based video coding, IEEE Trans. Circuit Syst. Video Technol., 9 (1): 156-160, February 1999.

[6] V.K. Srivastava and G.C. Ray, Design of 2D-multiple Notch Filter and Its Application in Reducing Blocking Artifact from DCT Coded Image, in Proc. 22nd Ann. IEEE Int. Conf. Engineering in Medicine and Biology, Chicago 2000: 2829-2833, July 2000.

[7] V.K. Srivastava, Post-processing of DCT coded images, $P h D$ dissertation, IIT Kanpur, India: Jan. 2001.

[8] H. Paek, R.-C. Kim, S.-U. Lee, A DCT-based spatially adaptive post processing technique to reduce the blocking artifacts in transform coded images, IEEE Trans. Circuits Syst. Video Technol. 10: 3641, February 2000.

[9] S. Minami, A. Zakhor, An optimization approach for removing blocking effects in transform coding, IEEE Trans. Circuits Syst. Video Technol., 5: 74-82, 1995.

[10] G. Lakhani, N. Zhong, Derivation of prediction equations for blocking effect reduction, IEEE Trans. Circuits Syst. Video Technol., 9: 415-418, 1999.

[11] G.A. Triantafyllidis, D. Tzovaras, M.G. Strintzis, Blocking artifact detection and reduction in compressed data, IEEE Trans. Circuits Syst. Video Technol., 12: 877-890, 2002.

[12] B. Jeon and J. Jeong, Blocking artifacts reduction in image compression with block boundary discontinuity criterion, IEEE Trans. Circuit Syst. Video Technol., 8 (3): 345-357, June 1998.

[13] Shih-Chang Hsia, Jar-Ferr Yang, and Bin-Da Liu, Efficient Postprocessor for Blocky Effect Removal Based on Transform Characteristics, IEEE Trans. Circuits Syst. Video Technol., 7 (6): 924-929, December 1997.

[14] T. Chen, H. R. Wu, and B. Qiu, "Adaptive postfiltering of transform coefficients for the reduction of blocking artifacts,” IEEE Trans. Circuits Syst. Video Technol., 11(5): 594-602, May 2001.

[15] S.-F. Chang and D. G. Messerschmitt, "Manipulation and compositing of MC-DCT compressed video,” IEEE J. Select. Areas Commun., 13(1): 1-11, Jan. 1995.

[16] S. Liu, A.C. Bovik, Efficient DCT-domain blind measurement and reduction of blocking artifacts, IEEE Trans. Circuits Syst. Video Technol. 12: 1139-1149, 2002.

[17] Y. Luo, R.K. Ward, Removing the blocking artifacts of block-based DCT compressed images, IEEE Trans. Image Process. 12 (7): 838-842, July 2003.

[18] Y. Zhao, G. Cheng and S. Yu, "Post-processing technique for blocking artifacts reduction in DCT domain”, Electronics Letters, 40 (19): 229-234, 16th September 2004.

[19] V.K. Srivastava, "A DCT based Algorithm for Blocking Artifact Reduction from DCT Coded Images”, in Proc. IEEE International Conference on Industrial Technology (ICIT2006): December 2006.

[20] V.K. Srivastava, "A SVD based scheme for Post-processing of DCT Coded Images”, in Proc. International Conference on Signal and Image Processing (IEEE ICSIP-06) at Hubli, Karnataka: December 2006.

[21] H. C. Andrews and C. L. Patterson, Singular value decompositions and digital image processing, IEEE Trans. Acoustics, Speech and Signal Processing, ASSP-24(1): 26-53, 1976.

[22] B. K. Natarajan, Filtering random noise from deterministic signals via data compression, IEEE Trans. on Signal Processing, 43(11): 2595-2605, 1995.

[23] K. Konstantinides , B. K. Natarajan, G. S. Yovanof, Noise estimation and filtering using block-based singular value decomposition, IEEE Trans. on Image Processing, 6(3): 479-483, 1997. 